\title{
Team Optimality Conditions of Differential Decision Systems with Nonclasssical Information Structures
}

\author{
Charalambos D. Charalambous, Themistoklis Charalambous and Christoforos N. Hadjicostis
}

\begin{abstract}
We derive team optimality conditions for differential decision systems with nonclassical information structures. The necessary conditions of optimality are given in terms of Hamiltonian system of equations consisting of a coupled backward and forward differential equations and a Hamiltonian projected onto the subspace generated by the information structures. Under certain global convexity conditions it is shown that Person-by-Person optimality implies team optimality.
\end{abstract}

\section{INTRODUCTION}

Over the years several articles have been written on decentralized decision making and information structures, and their applications in communication and queuing networks, sensor networks, and networked control systems (see, for example, [1]-[14] and references therein). The most popular mathematical formalism in the literature is that of "Static Team Theory developed by Marschak and Radner [15]-[17].

The main feature which differentiates classical decision making from decentralized decision making is the form of the information available at the control stations or DM to implement their actions, called "Information Structures or Patterns" [2]. Such information structures are called "Decentralized Information Structures" if the information available at control stations are not identical to all stations. Moreover, an information structure is called "Classical" if the following two conditions hold: i) all control stations have the same information structure, ii) all control stations have the property that whatever information structure is available at some time it is also available at any subsequent times, and it is called "Nonclassical" otherwise. A control station that has property ii) is said to have "Perfect Recall" [2], that is, its information structure is "Nested" in the sense that information is nondecreasing as time evolves.

In this paper, we draw the corresponding formulation for deterministic continuous- and discrete-time differential decision systems with nonclassical information structures, and we apply variational methods to derive team optimality conditions. The specific contributions of this paper are the following.

(a) Derive necessary and sufficient team optimality conditions, described in terms of the orthogonal projection of the Hamiltonian onto the closed Hilbert subspace generated by the nonclassical information structures;

C.D. Charalambous and C.N. Hadjicostis are with the Department of Electrical and Computer Engineering, University of Cyprus, Nicosia 1678 (E-mails: $\{$ chadcha, chadjic\}@ucy.ac.cy).

T. Charalambous is with with the Automatic Control Lab, Electrical Engineering Department and ACCESS Linnaeus Center, Royal Institute of Technology (KTH), Stockholm, Sweden. Corresponding author's address: Osquldas väg 10, 100-44 Stockholm, Sweden (E-mail: themisc@kth.se). (b) Show that under certain global convexity conditions on the Hamiltonian and the terminal pay-off, that Peson-byPerson $(\mathrm{PbP})$ optimality implies team optimality;

(c) Apply the optimality conditions to certain types of differential team problems.

This paper compliments our recent stochastic team optimality conditions described in terms of conditional expectation of the Hamiltonian onto the $\sigma$-algebra generated by the nonclassical information structures described in [14].

\section{NOTATION AND PRELIMINARIES}

The sets of real, integer and natural numbers are denoted by $\mathbb{R}, \mathbb{Z}$ and $\mathbb{N}$, respectively; $\mathbb{Z}_{N} \triangleq\{1,2, \ldots, N\}$ and $\mathbb{Z}_{N}^{0} \triangleq$ $\{0,1,2, \ldots, N\}$. The Borel algebra on $[0, T]$ is denoted by $\mathcal{B}([0, T])$ and the linear transformation mapping of a vector space $\mathcal{X}$ into a vector space $\mathcal{Y}$ is denoted by $\mathcal{L}(\mathcal{X}, \mathcal{Y}) .\langle a, b\rangle$ represents the inner product in $\mathbb{R}^{n}, \forall a, b \in \mathbb{R}^{n}$ for some positive integer $n$, whereas $|a|_{\mathbb{R}^{n}} \triangleq \sqrt{\langle a, a\rangle}$ is the norm on $\mathbb{R}^{n}, \forall a \in \mathbb{R}^{n}$ for some positive integer $n . \mathcal{H}=M \bigoplus M^{\perp}$ is a direct sum representation of a Hilbert space $\mathcal{H}$, where $M$ is a closed subspace of $\mathcal{H}$ and $M^{\perp}$ its orthogonal complement. $\boldsymbol{\Pi}_{M}(x)$ is the orthogonal projection of a Hilbert space element $x \in \mathcal{H}$ onto the subspace $M \subset \mathcal{H}$.

Our derivations will make use of the following spaces. $C\left([0, T], \mathbb{R}^{n}\right) \triangleq\{$ continuous functions $\phi:[0, T] \longrightarrow$ $\left.\mathbb{R}^{n}: \sup _{t \in[0, T]}|\phi(t)|_{\mathbb{R}^{n}}<\infty\right\} ; B^{\infty}\left([0, T], \mathbb{R}^{n}\right) \triangleq$ $\left\{\right.$ measurable functions $\phi:[0, T] \longrightarrow \mathbb{R}^{n}:\|\phi\|^{2} \triangleq$ $\left.\sup _{t \in[0, T]}|\phi(t)|_{\mathbb{R}^{n}}^{2}<\infty\right\}$. For Lebesgue measurable functions $\phi$ we have the following spaces: $L^{2}\left([0, T], \mathbb{R}^{n}\right) \triangleq$ $\left\{\phi:[0, T] \longrightarrow \mathbb{R}^{n}: \int_{[0, T]}|z(t)|_{\mathbb{R}^{n}}^{2} d t<\infty\right\}$, $L^{2}\left([0, T], \mathcal{L}\left(\mathbb{R}^{m}, \mathbb{R}^{n}\right)\right) \triangleq\left\{\phi:[0, T] \rightarrow \mathbb{R}^{n \times m}:\right.$ $\left.\int_{[0, T]}|\Sigma(t)|_{\mathcal{L}\left(\mathbb{R}^{m}, \mathbb{R}^{n}\right)}^{2} d t \triangleq \int_{[0, T]} \operatorname{tr}\left(\Sigma^{*}(t) \Sigma(t)\right) d t<\infty\right\}$.

\section{Team Problems of Differential Systems}

We first introduce the mathematical formulation of the team and person-by-person $(\mathrm{PbP})$ optimality of differential systems, and then we derive the optimality conditions.

\section{A. Elements of Team Games}

The basic elements of a team problem are the state space, the observation space, the DMs action spaces, and the pay-off. These are described below. 


\section{Unobserved State Space}

The unobserved state space is assumed to be a linear complete separable metric space $\left(\mathbb{X}_{[0, T]}, d\right)$, where $\mathcal{B}\left(\mathbb{X}_{[0, T]}\right)$ are the measurable subsets of the unobserved state space $\mathbb{X}_{[0, T]}$ generated by open sets (with respect to metric $d$ ). Elements $x \in \mathbb{X}_{[0, T]}$ are the unobserved state trajectories. Since state trajectories are solutions of differential equations, an envisioned scenario is $\mathbb{X}_{[0, T]}=C\left([0, T], \mathbb{R}^{n}\right)$, where $\left.\mathcal{B}\left(\mathcal{X}_{[0, T]}\right]\right)=\mathcal{B}\left(C\left([0, T], \mathbb{R}^{n}\right)\right)$ is the $\sigma$-field generated by cylinder sets in $C\left([0, T], \mathbb{R}^{n}\right)$, and a state trajectory is $x \triangleq\{x(t): t \in[0, T]\} \in C\left([0, T], \mathbb{R}^{n}\right)$. We also introduce $\sigma$-field generated by truncations of $x \in C\left([0, T], \mathbb{R}^{n}\right)$ defined by

$$
\begin{gathered}
\left.\mathcal{B}_{t}\left(C[0, T], \mathbb{R}^{n}\right)\right) \triangleq \sigma\left\{\left\{x \in C\left([0, T], \mathbb{R}^{m}\right): x(s) \in A\right\}:\right. \\
\left.0 \leq s \leq t, \quad A \in \mathcal{B}\left(\mathbb{R}^{n}\right)\right\}, t \in[0, T] .
\end{gathered}
$$

Thus, $\left.\left\{\mathcal{B}_{t}\left(C[0, T], \mathbb{R}^{n}\right)\right): t \in[0, T]\right\}$ is a family of $\sigma$-fields which is nondecreasing, $\mathcal{B}_{s}\left(C\left([0, T], \mathbb{R}^{n}\right)\right) \subseteq$ $\left.\mathcal{B}_{t}\left(C[0, T], \mathbb{R}^{n}\right)\right), 0 \leq s \leq t \leq T$. Thus, for continuous trajectories the space $C\left([0, T], \mathbb{R}^{n}\right)$ represent the unobserved state space, and its elements the unobserved state trajectories.

\section{Observation Space}

The observation space is assumed to be a linear complete separable metric space $\left(\mathbb{Y}_{[0, T]}^{i}, d^{i}\right)$, where $\mathcal{B}\left(\mathcal{Y}_{[0, T]}^{i}\right)$ are its measurable subsets generated by open sets, for $i=$ $1, \ldots, N$. Thus, elements $y \in \mathbb{Y}_{[0, T]}^{i}$ represent the observable trajectories. For unobserved state space $C\left([0, T], \mathbb{R}^{n}\right)$, the observable trajectories are generated by the maps

$$
\begin{gathered}
h^{i}:[0, T] \times C\left([0, T], \mathbb{R}^{n}\right) \longrightarrow \mathbb{R}^{k_{i}}, y^{i}(t) \triangleq h^{i}(t, x), \\
i=1, \ldots N,
\end{gathered}
$$

such that $\left\{h^{i}(t, x):(t, x) \in[0, T] \times C\left([0, T], \mathbb{R}^{n}\right)\right\}$ have the following property: for all $t \in[0, T]$, the map $(s, x) \rightarrow$ $h^{i}(s, x)$ is $\mathcal{B}([0, t]) \otimes \mathcal{B}_{t}\left(C\left([0, T], \mathbb{R}^{n}\right)\right) / \mathcal{B}\left(\mathbb{R}^{k_{i}}\right)$-measurable for $i=1, \ldots, N$. When this property holds we say, $\left\{y^{i}(t): t \in[0, T]\right\}$ is progressively measurable with respect to the family $\left.\left\{\mathcal{B}_{t}\left(C[0, T], \mathbb{R}^{k_{i}}\right)\right): t \in[0, T]\right\}$. Often we shall assume observation trajectories which are square integrable $y^{i} \in L^{2}\left([0, T], \mathbb{R}^{k_{i}}\right)$, and progressively measurable with respect to $\left.\left\{\mathcal{B}_{t}\left(C[0, T], \mathbb{R}^{k_{i}}\right)\right): t \in[0, T]\right\}$. Given an underlying Hilbert space $\mathcal{H}^{i}$ we denote by $\mathcal{H}_{0, t}^{y^{i}} \triangleq \overline{\operatorname{Span}}\left\{y^{i}(s): 0 \leq s \leq t\right\}$ the closed subspace generated by $\left\{y^{i}(s): 0 \leq s \leq t\right\}$ which is an element of the Hilbert space (i.e., $\mathcal{H}_{0, t}^{y^{i}} \subset \mathcal{H}^{i}$ ), $t \in[0, T]$, for $i=1, \ldots, N$ ). Note that the above constructions also embeds as a special case observation trajectories which are independent of $x$, by setting $y^{i}(t)=h^{i}(t), h^{i}:[0, T] \longrightarrow \mathbb{R}^{k_{i}}$, for $i=1, \ldots, N$.

\section{Team Members}

The team is assumed to consist of $N$ Decision Makers (DM) or players whose actions $\left\{u_{t}^{i}: t \in[0, T]\right\}$, take values in a closed convex set $\mathbb{A}^{i}$ of linear separable metric space $\left(\mathbb{M}^{i}, d^{i}\right), i=1, \ldots, N$. Unlike the centralized decision making, each DMs $i$ actions depends only on his own observation space $\left(\mathbb{Y}_{[0, T]}^{i}, d\right)$. Let $\left\{\mathcal{B}_{t}\left(\mathcal{Y}_{[0, T]}^{i}\right): t \in[0, T]\right\}$ denote the family of $\sigma$-fields generated by truncations of $y^{i} \in \mathbb{Y}_{[0, T]}^{i}, i=1, \ldots N$. The set of admissible laws or strategies of DM $i$, denoted by $\mathbb{U}^{i}[0, T]$, is defined by ${ }^{1}$

$$
\begin{gathered}
\mathbb{U}^{i}[0, T] \triangleq\left\{u^{i} \in L^{2}\left([0, T], \mathbb{R}^{d_{i}}\right): u_{t}^{i} \in \mathbb{A}^{i} \subset \mathbb{R}^{d_{i}},\right. \\
t \in[0, T], u^{i} \text { is }\left\{\mathcal{B}_{t}\left(\mathcal{Y}_{[0, T]}^{i}\right): t \in[0, T]\right\} \\
\text { - progressively measurable }\}, \quad \forall i \in \mathbb{Z}_{N} .
\end{gathered}
$$

Clearly, $\mathbb{U}^{i}[0, T]$ is a closed convex subset of $L_{\mathcal{Y}_{T}^{i}}^{2}\left([0, T], \mathbb{R}^{d_{i}}\right)$, for $i=1,2, \ldots, N$. The set of admissible $N$ team or person-by-person strategies is denoted by $\mathbb{U}^{(N)}[0, T] \triangleq \times_{i=1}^{N} \mathbb{U}^{i}[0, T]$. Clearly, such strategies are nonclassical because each member has different information structures.

The DM actions $\left\{u_{t}^{i}: t \in[0, T]\right\}$ are called:

Open Loop (OL), if $u_{t}^{i}=\mu^{i}(t)$, for $t \in[0, T]$, where $\mu^{i}:[0, T] \longrightarrow \mathbb{A}^{i}$ are deterministic measurable functions, $i=1, \ldots, N$;

Closed Loop Feedback (CLF), if $u_{t}^{i}=\mu^{i}\left(t, y^{i}\right)$ are nonanticipative functionals of the observation trajectory $y^{i}(\cdot)$, for $t \in[0, T]$, where $\mu^{i}:[0, T] \times \mathbb{Y}_{i[0, T]}^{i} \longrightarrow \mathbb{A}^{i}$, are deterministic measurable mappings, $i=1, \ldots, N$;

Closed Loop Memoryless (CLM), if $u_{t}^{i}=\mu^{i}\left(t, y^{i}(t)\right)$, for $t \in[0, T]$, where $\mu^{i}:[0, T] \times \mathbb{R}^{k_{i}} \longrightarrow \mathbb{A}^{i}$, are deterministic measurable functions, $i=1, \ldots, N$.

Note that open loop and closed loop feedback strategies are nonclassical because each team member has a different information structure, while closed loop memoryless strategies are nonnested. Clearly, open loop strategies can be described via observations $\left\{y^{i}(t): t \in[0, T]\right\}$ which belong to closed subspaces generated by finite number of basis, $\mathcal{H}_{0, t}^{y^{i}} \triangleq \operatorname{Span}\left\{e_{1}^{1}, e_{2}^{i}, \ldots, e_{j_{i}}^{i}\right\}$ of a Hilbert space $\mathcal{H}_{0, t}^{i}, t \in[0, T]$, for $i=1, \ldots, N$.

\section{Differential System}

A distributed differential system consists of an interconnection of $N$ subsystems, represented by a directed graph $\mathcal{G}$. Each subsystem $i$ has its own state vector $\mathbb{R}^{n_{i}}$, action space $\mathbb{A}^{i} \subset \mathbb{R}^{d_{i}}$, and an initial state vector $x^{i}(0)=x_{0}^{i}$, described by a system of coupled differential equations as follows.

$$
\begin{aligned}
& \dot{x}^{i}(t)=f^{i}\left(t, x^{i}(t), u_{t}^{i}\right) \\
& \quad+\sum_{j \in \mathcal{N}_{i}} f^{i j}\left(t, x^{j_{1}}(t), \ldots, x^{j_{\left|N_{i}\right|}}(t), u_{t}^{j_{1}}, \ldots, u_{t}^{j_{\left|N_{i}\right|}}\right), \\
& x^{i}(0)=x_{0}^{i}, \quad t \in(0, T], \quad i \in \mathbb{Z}_{N},
\end{aligned}
$$

where $\mathcal{N}_{i} \triangleq\left\{j_{1}, j_{2}, \ldots, j_{\left|N_{i}\right|}\right\}$ denotes the neighbors of subsystem $i$ with which there is coupling. Define the augmented vectors by

$u \triangleq\left(u^{1}, u^{2}, \ldots, u^{N}\right) \in \mathbb{R}^{d}, \quad x \triangleq\left(x^{1}, x^{2}, \ldots, x^{N}\right) \in \mathbb{R}^{n}$.

\footnotetext{
${ }^{1}$ We often write $L_{\mathcal{Y}_{T}^{i}}^{2}\left([0, T], \mathbb{R}^{d_{i}}\right) \equiv L^{2}\left([0, T], \mathbb{R}^{d_{i}}\right)$ to indicate that its elements are $\left\{\mathcal{B}_{t}\left(\mathcal{Y}_{[0, T]}^{i}\right): t \in[0, T]\right\}$-progressively measurable.
} 
In compact form the distributed differential system is described by

$$
\dot{x}(t)=f\left(t, x(t), u_{t}\right), \quad x(0)=x_{0}, \quad t \in(0, T],
$$

where $f:[0, T] \times \mathbb{R}^{n} \times \mathbb{A}^{(N)} \longrightarrow \mathbb{R}^{n}$. Note that (5) is very general since no specific interconnection structure is assumed among the different subsystems.

\section{Team and Person-by-Person Optimality}

Consider the distributed system (5) with a given admissible set of DMs strategies. Given a $u \in \mathbb{U}^{(N)}[0, T]$, we define the reward or performance criterion by

$$
J\left(u^{1}, \ldots, u^{N}\right) \triangleq \int_{0}^{T} \ell\left(t, x(t), u_{t}\right) d t+\varphi(x(T)),
$$

where $\ell:[0, T] \times \mathbb{R}^{n} \times \mathbb{U}^{(N)} \longrightarrow(-\infty, \infty]$ and $\varphi: \mathbb{R}^{n} \longrightarrow$ $(-\infty, \infty]$. Notice that the performance of the decentralized strategies is measured by a single pay-off functional. The underlying assumption concerning the single pay-off instead of multiple pay-offs (one for each decision maker) is that the team objective can be met.

Given the basic elements of the team game introduce above, we now introduce the definitions of team and Person-by-Person (PbP) or (player-by-player) optimality.

Problem 1: (Team and Person-by-Person Optimality) (T): Team Optimality. Given the pay-off functional (6), constraint (5) the $N$ tuple of strategies $u^{o} \triangleq$ $\left(u^{1, o}, u^{2, o}, \ldots, u^{N, o}\right) \in \mathbb{U}_{\text {reg }}^{(N)}[0, T]$ is called team optimal if it satisfies

$$
J\left(u^{1, o}, u^{2, o}, \ldots, u^{N, o}\right) \leq J\left(u^{1}, u^{2}, \ldots, u^{N}\right),
$$

for all $u \triangleq\left(u^{1}, u^{2}, \ldots, u^{N}\right) \in \mathbb{U}^{(N)}[0, T]$. Any $u^{o} \in$ $\mathbb{U}^{(N)}[0, T]$ satisfying (7) is called an optimal regular decision strategy (or control) and the corresponding $x^{o}(\cdot) \equiv$ $x\left(\cdot ; u^{o}(\cdot)\right)$ (satisfying (5)) the optimal state process.

(PbP): Person-by-Person Optimality. Given the pay-off functional (6), constraint (5) the $N$ tuple of strategies $u^{o} \triangleq\left(u^{1, o}, u^{2, o}, \ldots, u^{N, o}\right) \in \mathbb{U}^{(N)}[0, T]$ is called personby-person optimal if it satisfies

$$
\tilde{J}\left(u^{i, o}, u^{-i, o}\right)=J\left(u^{o}\right) \leq \tilde{J}\left(u^{i}, u^{-i, o}\right),
$$

for all $u^{i} \in \mathbb{U}^{i}[0, T], \quad \forall i \in \mathbb{Z}_{N}$, where

$$
\tilde{J}\left(v, u^{-i}\right) \triangleq J\left(u^{1}, u^{2}, \ldots, u^{i-1}, v, u^{i+1}, \ldots, u^{N}\right) .
$$

Conditions (8) are analogous to the Nash equilibrium strategies consisting of a single pay-off. However, the information structures are nonclassical.

\section{B. Existence of Solutions and Continuous Dependence}

Herein, we study the question of existence of solutions to (5) and its continuous dependence on the DM strategies.

Assumptions 1: The drift $f$ associated with (5) is a Borel measurable map defined by

$$
f:[0, T] \times \mathbb{R}^{n} \times \mathbb{A}^{(N)} \longrightarrow \mathbb{R}^{n},
$$

and there exists a $K \in L^{2,+}([0, T], \mathbb{R})$ such that (A1) $|f(t, x, u)-f(t, y, u)|_{\mathbb{R}^{n}} \leq K(t)|x-y|_{\mathbb{R}^{n}}$ uniformly in $u \in \mathbb{A}^{(N)}$;

(A2) $|f(t, x, u)-f(t, x, v)|_{\mathbb{R}^{n}} \leq K(t)|u-v|_{\mathbb{R}^{d}}$ uniformly in $x \in \mathbb{R}^{n}$;

(A3) $|f(t, x, u)|_{\mathbb{R}^{n}} \leq K(t)\left(1+|x|_{\mathbb{R}^{n}}\right)$ uniformly in $u \in$ $\mathbb{A}^{(N)}$;

(A4) For any $x, \tilde{x} \in C\left([0, T], \mathbb{R}^{n}\right),\left|h^{i}(t, x)-h^{i}(t, \tilde{x})\right|_{\mathbb{R}^{k_{i}}} \leq$ $K|x-\tilde{x}|_{C\left([0, T], \mathbb{R}^{n}\right)}, K>0, i=1, \ldots, N$;

Assumptions 1 are sufficient conditions for the existence of a unique $C\left([0, T], \mathbb{R}^{n}\right)$ solution such that $B^{\infty}\left([0, T], \mathbb{R}^{n}\right)$. The following lemma establishes such results and continuous dependence of solutions on the DM strategies.

Lemma 1: Suppose Assumptions 1 hold. Then for any $u \in$ $\mathbb{U}^{(N)}[0, T]$, the following hold.

1) System (5) has a unique solution $x \in B^{\infty}\left([0, T], \mathbb{R}^{n}\right)$ which is continuous $x \in C\left([0, T], \mathbb{R}^{n}\right)$.

2) The solution of system (5) is continuously dependent on the DM strategies, in the sense that, as $u^{i, \alpha} \longrightarrow u^{i, o}$ in $\mathbb{U}^{i}[0, T], \forall i \in \mathbb{Z}_{N}, x^{\alpha} \longrightarrow x^{o}$ in $B^{\infty}\left([0, T], \mathbb{R}^{n}\right)$.

Proof: Similar to [18, Lemma 1].

\section{Team and PbP Optimality Conditions}

For the derivation of optimality conditions we shall require the stronger regularity conditions given below.

Assumptions 2: The maps of $\{f, \ell, \varphi\}$ satisfy the following conditions.

(B1) The map $f:[0, T] \times \mathbb{R}^{n} \times \mathbb{A}^{(N)} \longrightarrow \mathbb{R}^{n}$ is continuous in $(t, x, u)$ and continously differentiable with respect to $x, u$; (B2) The first derivatives of $\left\{f_{x}, f_{u}\right\}$ are bounded uniformly on $[0, T] \times \mathbb{R}^{n} \times \mathbb{A}^{(N)}$.

(B3) The maps $\ell:[0, T] \times \mathbb{R}^{n} \times \mathbb{A}^{(N)} \longrightarrow(-\infty, \infty]$ is Borel measurable, continuously differentiable with respect to $(x, u)$, the map $\varphi:[0, T] \times \mathbb{R}^{n} \longrightarrow(-\infty, \infty]$ is continously differentiable with respect to $x, \ell(t, 0,0)$ is bounded, and there exist $K_{1}, K_{2}>0$ such that

$$
\begin{aligned}
\left|\ell_{x}(t, x, u)\right|_{\mathbb{R}^{n}}+\left|\ell_{u}(t, x, u)\right|_{\mathbb{R}^{d}} & \leq K_{1}\left(1+|x|_{\mathbb{R}^{n}}+|u|_{\mathbb{R}^{d}}\right), \\
\left|\varphi_{x}(x)\right|_{\mathbb{R}^{n}} & \leq K_{2}\left(1+|x|_{\mathbb{R}^{n}}\right) .
\end{aligned}
$$

(B4) $\left|h^{i}(t, x)\right|_{\mathbb{R}^{k_{i}}} \leq K \sup _{0 \leq s \leq t}\left(1+|x(s)|_{\mathbb{R}^{n}}^{2}\right), \forall t \in$ $[0, T], x \in C\left([0, T], \mathbb{R}^{n}\right), i=1, \ldots, N$.

Note that (B1), (B2) imply that $|f(t, x, u)|_{\mathbb{R}^{n}} \leq$ $K\left(1+|x|_{\mathbb{R}^{n}}+|u|_{\mathbb{R}^{d}}\right), K>0$, and (B4) implies $h^{i} \in B^{\infty}\left([0, T], \mathbb{R}^{n}\right), i=1, \ldots, N$.

First, we derive necessary conditions for team and $\mathrm{PbP}$ optimality. For this derivation, we need the so-called variational equation. We note that for differential systems, the strategies can be either open-loop or feedback, and feedback strategies do not give smaller pay-off. Thus, the minimum pay-off attainable under open loop strategies is equal to the minimum pay-off attainable under feedback strategies. This is well known in deterministic optimal control theory. The point to be made is that when considering variations in the state trajectory the DM strategies do not react so we do not 
need to introduce derivatives of the $u$ variable with respect to the state.

Suppose $u^{o} \triangleq\left(u^{1, o}, u^{2, o}, \ldots, u^{N, o}\right) \in \mathbb{U}^{(N)}[0, T]$ denotes the optimal decision and $u \triangleq\left(u^{1}, u^{2}, \ldots, u^{N}\right) \in \mathbb{U}^{(N)}[0, T]$ any other decision. Since $\mathbb{A}^{i}$ is convex then $\mathbb{U}^{i}[0, T]$ is convex $\forall i \in \mathbb{Z}_{N}$, it is clear that for any $\varepsilon \in[0,1]$,

$$
u_{t}^{i, \varepsilon} \triangleq u_{t}^{i, o}+\varepsilon\left(u_{t}^{i}-u_{t}^{i, o}\right) \in \mathbb{U}^{i}[0, T], \quad \forall i \in \mathbb{Z}_{N} .
$$

Let $x^{\varepsilon}(\cdot) \equiv x^{\varepsilon}\left(\cdot ; u^{\varepsilon}(\cdot)\right)$ and $x^{o}(\cdot) \equiv x^{o}\left(\cdot ; u^{o}(\cdot)\right) \in$ $B^{\infty}\left([0, T], \mathbb{R}^{n}\right)$ denote the solutions of the system equation (5) corresponding to $u^{\varepsilon}(\cdot)$ and $u^{o}(\cdot)$, respectively. Consider the limit

$$
Z(t) \triangleq \lim _{\varepsilon \downarrow 0} \frac{1}{\varepsilon}\left\{x^{\varepsilon}(t)-x^{o}(t)\right\}, \quad t \in[0, T] .
$$

We have the following result characterizing the variational equation.

Lemma 2: Suppose Assumptions 2 hold and consider strategies $\mathbb{U}^{(N)}[0, T]$. The process $\{Z(t): t \in[0, T]\}$ defined by (9) is an element of the Banach space $B^{\infty}\left([0, T], \mathbb{R}^{n}\right)$ and it is the unique solution of the variational differential equation

$$
\begin{aligned}
\dot{Z}(t)= & f_{x}\left(t, x^{o}(t), u_{t}^{o}\right) Z(t) \\
& +\sum_{i=1}^{N} f_{u^{i}}\left(t, x^{o}(t), u_{t}^{o}\right)\left(u_{t}^{i}-u_{t}^{i, o}\right), Z(0)=0 .
\end{aligned}
$$

having trajectories $Z \in C\left([0, T], \mathbb{R}^{n}\right)$.

Proof: Similar to [18, Lemma 2].

Before we show the optimality conditions we define the Hamiltonian system of equations, i.e.,

$$
\begin{aligned}
& H:[0, T] \times \mathbb{R}^{n} \times \mathbb{R}^{n} \times \mathbb{A}^{(N)} \longrightarrow \mathbb{R}, \\
& H(t, x, \psi, u) \triangleq\langle f(t, x, u), \psi\rangle+\ell(t, x, u) .
\end{aligned}
$$

For any $u \in \mathbb{U}^{(N)}[0, T]$, the adjoint process $\psi \in$ $L^{2}\left([0, T], \mathbb{R}^{n}\right)$ satisfies the following backward differential equation

$$
\begin{aligned}
\dot{\psi}(t) & =-f_{x}^{*}\left(t, x(t), u_{t}\right) \psi(t)-\ell_{x}\left(t, x(t), u_{t}\right) \\
& =-H_{x}\left(t, x(t), \psi(t), u_{t}\right), \quad t \in[0, T) . \\
\psi(T) & =\varphi_{x}(x(T)) .
\end{aligned}
$$

In terms of the Hamiltonian, the state process satisfies the differential equation

$$
\begin{aligned}
& \dot{x}(t)=f\left(t, x(t), u_{t}\right)=H_{\psi}\left(t, x(t), \psi(t), u_{t}\right), t \in(0, T] \\
& x(0)=x_{0} .
\end{aligned}
$$

Next, we state and prove the necessary conditions for team optimality.

Theorem 1 (Necessary conditions for team optimality):

Consider Problem 1 under Assumptions 2, and assume $\mathbb{A}^{i}$ are closed, bounded and convex subsets of $\mathbb{R}^{d_{i}}$, and $\left\{y^{i}(s): 0 \leq s \leq t\right\}$ generates $\mathcal{H}_{0, t^{i}}^{y^{i}}$ a closed subspace of a Hilbert space for $i=1, \ldots, N$.

For an element $u^{o} \in \mathbb{U}^{(N)}[0, T]$ with the corresponding solution $x^{o} \in B^{\infty}\left([0, T], \mathbb{R}^{n}\right)$ to be team optimal, it is necessary that the following conditions hold.

1) There exists a process $\psi^{o} \in L^{2}\left([0, T], \mathbb{R}^{n}\right)$.

2) The triple $\left\{u^{o}, x^{o}, \psi^{o}\right\}$ satisfy the inequality:

$$
\begin{gathered}
\sum_{i=1}^{N} \int_{0}^{T}\left\langle H_{u^{i}}\left(t, x^{o}(t) \psi^{o}(t), u_{t}^{o}\right), u_{t}^{i}-u_{t}^{i, o}\right\rangle d t \geq 0, \\
\forall u \in \mathbb{U}^{(N)}[0, T] .
\end{gathered}
$$

3) The process $\psi^{o}$ is the unique $C\left([0, T], \mathbb{R}^{n}\right)$ solution of the backward differential equation (12a), (12b) and $u^{o} \in$ $\mathbb{U}^{(N)}[0, T]$ satisfies the inequalities:

$$
\begin{gathered}
\left\langle\boldsymbol{\Pi}_{\mathcal{H}_{0, t}^{y^{i}}}\left(H_{u^{i}}\left(t, x^{o}(t), \psi^{o}(t), u_{t}^{o}\right)\right), v^{i}-u_{t}^{i, o}\right\rangle \geq 0, \\
\forall v^{i} \in \mathbb{A}^{i}, t \in[0, T], i=1,2, \ldots, N .
\end{gathered}
$$

Proof: For 1) and 2), this is similar to [18, Theorem 6]. For 3), consider $\left\{H_{u^{i}}\left(t, x^{o}, \psi^{o}(t), u_{t}^{o}\right): t \in[0, T]\right\}$ lying in the Hilbert space of square integrable functions $\mathcal{H}^{i}, i=$ $1, \ldots, N$, and the set of observables $\left\{y^{i}(t): t \in[0, T]\right\}$ generating a closed subspace $\mathcal{H}_{0, t}^{y^{i}} \triangleq \overline{\operatorname{Span}}\left\{y^{i}(s): 0 \leq s \leq\right.$ t\} $\subset \mathcal{H}^{i}, i \in \mathbb{Z}_{N}$. Then for any $H_{u^{i}} \in \mathcal{H}^{i}$ we have the decomposition

$$
\begin{aligned}
& H_{u^{i}}\left(t, x^{o}(t), \psi^{o}(t), u_{t}^{o}\right) \\
& \quad=\Pi_{\mathcal{H}_{0, t}^{y^{i}}}\left(H_{u^{i}}\left(t, x^{o}(t), \psi^{o}(t), u_{t}^{o}\right)\right)+E(t), \\
& \quad E(t) \perp \mathcal{H}_{0, t}^{y^{i}}, t \in[0, T], i \in \mathbb{Z}_{N} .
\end{aligned}
$$

Since $u_{t}^{i}-u_{t}^{i, o} \in \mathcal{H}_{0, t}^{y^{i}}$, by substituting the above decomposition in (13) we obtain

$$
\begin{aligned}
\sum_{i=1}^{N} \int_{0}^{T}\left\langle\boldsymbol{\Pi}_{\mathcal{H}_{0, t}^{y^{i}}}\left(H_{u^{i}}\left(t, x^{o}(t), \psi^{o}(t), u_{t}^{o}\right)\right),\right. \\
\left.u_{t}^{i}-u_{t}^{i, o}\right\rangle d t \geq 0, \quad \forall u \in \mathbb{U}^{(N)}[0, T] .
\end{aligned}
$$

Let $t \in(0, T)$, and $\varepsilon>0$, and consider the set $I_{\varepsilon} \equiv[t, t+$ $\varepsilon] \subset[0, T]$ such that $\left|I_{\varepsilon}\right| \rightarrow 0$ as $\varepsilon \rightarrow 0$, for $i=1,2, \ldots, N$. For any $\mathcal{H}_{0, t}^{y^{i}}$-progressively measurable $v_{t}^{i} \in \mathbb{A}^{i}$, construct

$$
u_{t}^{i}=\left\{\begin{array}{ll}
v_{t}^{i} & \text { for } t \in I_{\varepsilon} \\
u_{t}^{i, o} & \text { otherwise }
\end{array} \quad i=1,2, \ldots, N .\right.
$$

Clearly, it follows from the above construction that $u^{i} \in$ $\mathbb{U}^{i}[0, T]$. Substituting (16) in (15) we obtain the following inequality

$$
\begin{gathered}
\left.\sum_{i=1}^{N} \int_{I_{\varepsilon}}\left\langle\boldsymbol{\Pi}_{\mathcal{H}_{0, t}^{y^{i}}}\left(H_{u^{i}}\left(t, x^{o}(t), \psi^{o}(t), u_{t}^{o}\right)\right), v_{t}^{i}-u_{t}^{i, o}\right)\right\rangle d t \\
\geq 0, \forall v_{t}^{i} \in \mathbb{A}^{i}, \quad i=1,2, \ldots, N
\end{gathered}
$$

Letting $\left|I_{\varepsilon}\right|$ denote the Lebesgue measure of the set $I_{\varepsilon}$ and dividing the above expression by $\left|I_{\varepsilon}\right|$ and letting $\varepsilon \rightarrow 0$ we arrive at the following inequality.

$$
\begin{gathered}
\sum_{i=1}^{N}\left\langle\boldsymbol{\Pi}_{\mathcal{H}_{0, t}^{y^{i}}}\left(\mathbb{H}_{u^{i}}\left(t, x^{o}(t), \psi^{o}(t), u_{t}^{o}\right), v_{t}^{i}-u_{t}^{i, o}\right\rangle \geq 0\right. \\
\forall v_{t}^{i} \in \mathbb{A}^{i}, \quad t \in[0, T], \quad i=1,2, \ldots, N
\end{gathered}
$$


To complete the proof of 3 ) for a given $v^{i} \in \mathbb{A}^{i}$ define

$$
\begin{gathered}
g^{i}(t) \triangleq\left\langle\boldsymbol{\Pi}_{\mathcal{H}_{0, t}^{y^{i}}}\left(\mathbb{H}_{u^{i}}\left(t, x^{o}(t), \psi^{o}(t), u_{t}^{o}\right), v^{i}-u_{t}^{i, o}\right\rangle,\right. \\
t \in[0, T], \quad i=1,2, \ldots, N .
\end{gathered}
$$

Then $g^{i}(t) \in \mathcal{H}_{0, t}^{y^{i}}$. We shall show that

$$
g^{i}(t) \geq 0, \quad \forall v^{i} \in \mathbb{A}^{i}, \quad t \in[0, T], \forall i \in \mathbb{Z}_{N} .
$$

Suppose for some $i \in \mathbb{Z}_{N}$, (20) does not hold, and let $A^{i} \triangleq$ $\left\{t: g^{i}(t)<0\right\}$. Since $g^{i}(t) \in \mathcal{H}_{0, t}^{y^{i}}, \forall t \in[0, T]$ we can choose $v_{t}^{i}$ in (18) as

$$
v_{t}^{i} \triangleq\left\{\begin{array}{l}
v \text { on } A^{i} \\
u_{t}^{i, o} \text { outside } A^{i}
\end{array}\right.
$$

together with $v_{t}^{j}=u_{t}^{j, o}, j \neq i, j \in \mathbb{Z}_{N}$. Substituting this in (15) (with $u_{t}^{i}=v_{t}^{i}$ ) we arrive at $\int_{A^{i}} g^{i}(t) d t \geq 0$, which contradicts the definition of $A^{i}$, unless $A^{i}$ has Lebesgue measure zero. Hence, (20) holds which is precisely (14). This completes the derivation.

Next, we show that the necessary conditions of optimality (14) are also sufficient under certain convexity conditions.

Theorem 2 (Sufficient conditions for team optimality):

Consider Problem 1 under the conditions of Theorem 1, and let $\left(u^{o}(\cdot), x^{o}(\cdot)\right)$ denote any control-state pair (decisionstate) and let $\psi^{o}(\cdot)$ the corresponding adjoint processes. Suppose the following conditions hold:

C1 $H(t, \cdot, x, u), t \in[0, T]$ is convex in $(x, u) \in \mathbb{R}^{n} \times \mathbb{A}^{(N)}$; C2 $\varphi(\cdot)$ is convex in $x \in \mathbb{R}^{n}$.

Then $\left(u^{o}(\cdot), x^{o}(\cdot)\right)$ is team optimal if it satisfies (14). In other words, necessary conditions are also sufficient.

Proof: Omitted due to space limitations.

Under the conditions of Theorem 1, it is easy to deduce that the necessary conditions for team optimality and $\mathrm{PbP}$ optimality are equivalent. Moreover, under the conditions of Theorem 2 it can be shown that PbP optimality implies team optimality. We state the results as a corollary.

Corollary 1: (Necessary and sufficient conditions for PbP optimality). Consider the PbP optimality of Problem 1 under the conditions of Theorem 1, 2 .

The necessary and sufficient conditions for $\mathrm{PbP}$ optimality of $\left(u^{o}(\cdot), x^{o}(\cdot)\right)$ are those of team optimality given in Theorems 1,2 with the variational inequality (13) replaced by

$$
\begin{gathered}
\int_{0}^{T}\left\langle\boldsymbol{\Pi}_{\mathcal{H}_{0, t}^{y^{i}}}\left(H_{u^{i}}\left(t, x^{o}(t), \psi^{o}(t), u_{t}^{o}\right)\right), u_{t}^{i}-u_{t}^{i, o}\right\rangle d t \geq 0 \\
\forall u^{i} \in \mathbb{U}^{i}[0, T], \quad \forall i \in \mathbb{Z}_{N}
\end{gathered}
$$

Proof: Similar to that of Theorems 1 and 2.

We conclude this section by stating that the team optimality conditions, based on Pontryagin's maximum principle are obtained following the classical theory of deterministic optimal control with centralized strategies. The only variation is the characterization of the optimal strategies described by the projection of the Hamiltonian onto the Hilbert closed subspace generated by the observables (on which the different DM actions are based on). Consequently, we state following observations.
(O1): By considering spike or needle variations, condition, the derivatives of $f$ and $\ell$ w.r.t. $u$ can be removed and replaced by $f, \ell, \varphi$ that are twice differentiable in $x \in$ $\mathbb{R}^{n}$, having first partial derivatives which are measurable in $t \in[0, T]$ and continuous with respect to the rest of the arguments, and second partial derivatives which are uniformly bounded.

(O2): The team and PbP optimality conditions of Theorem 1, 2 are based on the assumption that $\mathbb{A}^{i}, i=1, \ldots, N$ are convex. We can consider relaxed strategies, that is, controls which are conditional distributions, $u_{t}^{i}\left(d \xi \mid\left\{y^{i}(s): 0 \leq\right.\right.$ $s \leq t\}), i=1, \ldots, N$, and remove the assumptions on the differentiability of $f, \ell$ with respect to $u$, and instead assume $\mathbb{A}^{i}, i=1, \ldots, N$ are compact subsets of finite-dimensional spaces. Based on this relaxed strategies formulation we can show existence of optimal strategies utilizing appropriate weak* topologies. Such relaxed strategies are important when the DM actions are based on a finite number of points, such as, $\mathbb{A}^{i}=\{-1,+1\}$ which is not a convex set.

(O3): The team and PbP optimality conditions of Theorem 1, 2 can be generalized to include pointwise and integral constraints, of $x, u$ involving inequalities and equalities. Moreover, the terminal time can be free laying on a manifold, and hence subject to optimization rather than been fixed $T$.

\section{EXAMPLES}

\section{A. Generalized Normal Form (GNF)}

Definition 1 (Generalized Normal Form): The game is said to have "general normal form" if

$$
\begin{aligned}
f(t, x, u) \triangleq & b(t, x)+g(t, x) u, \\
& g(t, x) u \triangleq \sum_{j=1}^{N} g^{(j)}(t, x) u^{j}, \\
\ell(t, x, u) \triangleq & \frac{1}{2}\langle u, R(t, x) u\rangle+\frac{1}{2} \lambda(t, x)+\langle u, \eta(t, x)\rangle,
\end{aligned}
$$

where

$$
\begin{aligned}
\langle u, R(t, x) u\rangle & \triangleq \sum_{i=1}^{N} \sum_{j=1}^{N} u^{i, *} R_{i j}(t, x) u^{j}, \\
\langle u, \eta(t, x)\rangle & \triangleq \sum_{i=1}^{N} u^{i, *} \eta^{i}(t, x)
\end{aligned}
$$

and $R(\cdot, \cdot)$ is symmetric uniformly positive definite, and $\lambda(\cdot, \cdot)$ is uniformly positive semidefinite.

GNF refers to the case when the drift coefficient $f$ is linear with respect to (w.r.t.) the decision variable $u$, and the payoff function $\ell$ is quadratic in $u$, while $f, \ell, \varphi$ are nonlinear with respect to $x$.

By the definition of Hamiltonian (11), its derivative is

$$
\begin{array}{r}
H_{u}(t, x, \psi, Q, u)=g^{*}(t, x) \psi+R(t, x) u+\eta(t, x), \\
(t, x) \in[0, T] \times \mathbb{R}^{n} .
\end{array}
$$


By Theorem 1, utilizing the fact that $u_{t}^{i, o} \in \mathcal{H}_{0, t}^{y^{i}}$ for each $i \in \mathbb{Z}_{N}$, the explicit expression for $u_{t}^{i, o}$ is given by

$$
\begin{aligned}
u_{t}^{i, o}= & -\left\{\boldsymbol{\Pi}_{\mathcal{H}_{0, t}^{y^{i}}}\left(R_{i i}\left(t, x^{o}(t), \psi_{x}^{o}(t)\right)\right)\right\}^{-1} \\
& \left\{\boldsymbol{\Pi}_{\mathcal{H}_{0, t}^{y^{i}}}\left(\eta^{i}\left(t, x^{o}(t)\right)\right)\right. \\
& +\sum_{j=1, j \neq i}^{N} \boldsymbol{\Pi}_{\mathcal{H}_{0, t}^{y^{i}}}\left(R_{i j}\left(t, x^{o}(t)\right) u_{t}^{j, o}\right) \\
& \left.+\boldsymbol{\Pi}_{\mathcal{H}_{0, t}^{y^{i}}}\left(g^{(i), *}(t, x) \psi^{o}(t)\right)\right\}, \quad i=1,2, \ldots, N .
\end{aligned}
$$

\section{B. Linear Quadratic Form (LQF)}

Definition 2 (Quadratic Form): The game is said to have "linear quadratic form" if

$$
\begin{aligned}
f(t, x, u)= & A(t) x+b(t)+B(t) u, \\
\ell(t, x)= & \frac{1}{2}\langle u, R(t) u\rangle+\frac{1}{2}\langle x, H(t) x\rangle+\langle x, F(t)\rangle \\
& +\langle u, E(t) x\rangle+\langle u, m(t)\rangle, \\
\varphi(x)= & \frac{1}{2}\langle x, M(T) x\rangle+\langle x, N(T)\rangle,
\end{aligned}
$$

and $R(\cdot)$ is symmetric uniformly positive definite, $H(\cdot)$ is symmetric uniformly positive semidefinite, and $M(T)$ is symmetric positive semidefinite.

From the optimal strategies under LQF, one obtains for $i=$ $1,2, \ldots, N$ :

$$
\begin{aligned}
& u_{t}^{i, o}=-\left\{R_{i i}(t)\right\}^{-1}\left\{m^{i}(t)+\sum_{j=1}^{N} E_{i j}(t) \boldsymbol{\Pi}_{\mathcal{H}_{0, t}^{y^{i}}}\left(x^{j, o}(t)\right)\right. \\
& \left.+\sum_{j=1, j \neq i}^{N} R_{i j} \boldsymbol{\Pi}_{\mathcal{H}_{0, t}^{y^{i}}}\left(u_{t}^{j, o}\right)+B^{(i), *}(t) \boldsymbol{\Pi}_{\mathcal{H}_{0, t}^{y^{i}}}\left(\psi^{o}(t)\right)\right\} .
\end{aligned}
$$

Note that the previous equations can be put in the form of fixed point matrix equation.

\section{DISCRETE-TIME DYNAMICAL SYSTEMS}

By either discretizing the continuous-time system (or considering the discrete-time analog), we have the Hamiltonian at each time step $k$ given by

$$
H(k, x, \psi, u) \triangleq\langle f(k, x, u), \psi(k+1)\rangle+\ell(k, x, u),
$$

where $k \in \mathbb{Z}_{T-1}^{0}$ and $T$ is a positive integer. Note that the adjoint is one step ahead of the other terms. In terms of the Hamiltonian, the state process satisfies

$$
\begin{aligned}
& \quad x(k+1)=f\left(k, x(k), u_{k}\right) \\
& \quad=H_{\psi}\left(k, x(k), \psi(k+1), u_{k}\right), k \in \mathbb{Z}_{T-1}^{0} \\
& x(0)=x_{0} .
\end{aligned}
$$

For any $u \in \mathbb{U}^{(N)}[0, T]$, the adjoint process is $\psi \in$ $\ell^{2}\left([0, T], \mathbb{R}^{n}\right)$ satisfies the backward differential equation

$$
\begin{array}{r}
\psi(k)=-H_{x}\left(k, x(k), \psi(k+1), u_{k}\right), k \in \mathbb{Z}_{T-1}^{0} . \\
\psi(T)=\varphi_{x}(x(T)) .
\end{array}
$$

The process $\psi^{\circ}$ is the unique solution of the backward difference equation (24a), (24b) and $u^{o} \in \mathbb{U}^{(N)}[0, T]$ satisfies the inequalities:

$$
\begin{gathered}
\left\langle\boldsymbol{\Pi}_{\mathcal{H}_{0, k}^{y^{i}}}\left(H_{u^{i}}\left(k, x^{o}(k), \psi^{o}(k+1), u_{k}^{o}\right)\right), v^{i}-u_{k}^{i, o}\right\rangle \geq 0 \\
\forall v^{i} \in \mathbb{A}^{i}, k \in[0, T], i=1,2, \ldots, N
\end{gathered}
$$

\section{REFERENCES}

[1] H. S. Witsenhausen, "A counter example in stochastic optimum control," SIAM Journal on Control and Optimization, vol. 6, no. 1, pp. 131-147, 1968.

[2] - "Separation of estimation and control for discrete time systems," in Proceedings of the IEEE, 1971, pp. 1557-1566.

[3] Y.-C. Ho and K.-C. Chu, "Team decision theory and information structures in optimal control problems-part I," IEEE Transactions on Automatic Control, vol. 17, no. 1, pp. 15-22, 1972.

[4] P. Varaiya and J. Walrand, "Decentralized stochastic control," University of California, Berkeley, Tech. Rep., 1977.

[5] A. Bagghi and T. Basar, "Teams decision theory for linear continuoustime systems," IEEE Transactions on Automatic Control, vol. 25, no. 6, pp. 1154-1161, 1980.

[6] J. Walrand and P. Varaiya, "Optimal causal coding-decoding problems," IEEE Transactions on Information Theory, vol. 29, no. 3, pp. 814-820, 1983.

[7] R. Bansar, "An equilibrium theory for multiperson decision making with multiple probabilistic models," IEEE Transactions on Automatic Control, vol. 30, no. 2, pp. 118-132, 19875.

[8] M. Aicardi, F. Davoli, and R. Minciardi, "Decentralized optimal control of markov chains with a common past information," IEEE Transactions on Automatic Control, vol. 32, no. 11, pp. 1028-1031, 1987.

[9] P. R. Wall and J. H. van Schuppen, "A class of team problems with discrete action spaces: Optimality conditions based on multimodularity," SIAM Journal on Control and Optimization, vol. 38, no. 3, pp. 875-892, 2000

[10] B. Bamieh and P. Voulgaris, "A convex characterization of distributed control problems in spatially invariant systems with communication constraints," Systems and Control Letters, vol. 54, no. 6, pp. 575-583, 2005.

[11] A. Mahajan and D. Teneketzis, "Optimal design of sequential real-time communicaton systems," IEEE Transactions on Information Theory, vol. 55, no. 11, pp. 5317-5337, 2009.

[12] A. Nayyar, A. Mahajan, and D. Teneketzis, "Optimal control strategies in delayed sharing information structures," IEEE Transactions on Automatic Control, vol. 56, no. 7, pp. 1606-1620, 2011.

[13] J. H. van Schuppen, O. Boutin, P. L. Kempker, J. Komenda, T. Masopust, N. Pambakian, and A. C. M. Ran, "Control of distributed systems: Tutorial and overview," European Journal on Control, pp. 579-602, 2012.

[14] C. D. Charalambous and N. U. Ahmed, "Dynamic team theory of stochastic differential decision systems with decentralized noisy information structures via Girsanov's measure transformation,' Submitted to Journal of Mathematics of Control, Signals and System, p. 56, October 2013. [Online]. Available: http://arxiv.org/abs/1309. 1913

[15] J. Marschak, "Elements for a theory of teams," Management Science, vol. 1 , no. 2,1955 .

[16] R. Radner, "Team decision problems," The Annals of Mathematical Statistics, vol. 33, no. 3, pp. 857-881, 1962.

[17] J. Marschak and R. Radner, Economic Theory of Teams. New Haven: Yale University Press, 1972.

[18] C. D. Charalambous and N. U. Ahmed, "Centralized versus decentralized optimization of distributed stochastic differential decision systems with noiseless information structures-Part I: General theory," Submitted to IEEE Transactions on Automatic Control, p. 39, February 2013. [Online]. Available: http://arxiv.org/abs/1302.3452 